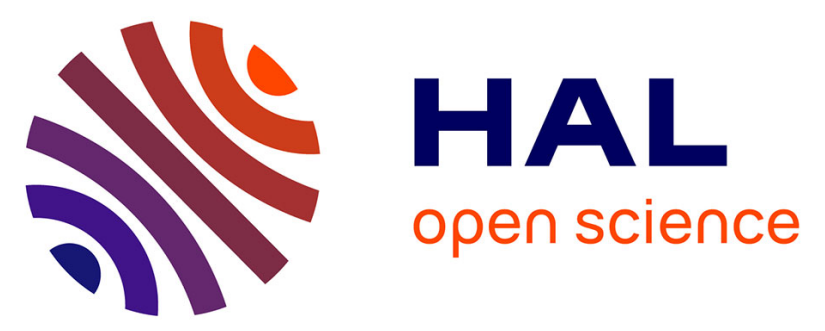

\title{
Control of Molecule-Metal Interaction by Hydrogen Manipulation in an Organic Molecule
}

Van Dong Pham, Vincent Repain, Cyril Chacon, Amandine Bellec, Yann

Girard, Sylvie Rousset, Alexander Smogunov, Yannick J. Dappe, Jerome Lagoute

\section{To cite this version:}

Van Dong Pham, Vincent Repain, Cyril Chacon, Amandine Bellec, Yann Girard, et al.. Control of Molecule-Metal Interaction by Hydrogen Manipulation in an Organic Molecule. Journal of Physical Chemistry Letters, 2016, 7 (8), pp.1416 - 1421. 10.1021/acs.jpclett.6b00476 . cea-01485682

\section{HAL Id: cea-01485682 https://hal-cea.archives-ouvertes.fr/cea-01485682}

Submitted on 9 Mar 2017

HAL is a multi-disciplinary open access archive for the deposit and dissemination of scientific research documents, whether they are published or not. The documents may come from teaching and research institutions in France or abroad, or from public or private research centers.
L'archive ouverte pluridisciplinaire HAL, est destinée au dépôt et à la diffusion de documents scientifiques de niveau recherche, publiés ou non, émanant des établissements d'enseignement et de recherche français ou étrangers, des laboratoires publics ou privés. 


\title{
Control of Molecule-Metal Interaction by Hydrogen Manipulation in an Organic Molecule
}

\author{
Van Dong Pham, ${ }^{\dagger}$ Vincent Repain, $^{\dagger}$ Cyril Chacon, ${ }^{\dagger}$ Amandine Bellec, $^{\dagger}$ Yann Girard, ${ }^{\dagger}$ Sylvie Rousset, ${ }^{\dagger}$ \\ Alexander Smogunov, ${ }^{\ddagger}$ Yannick J. Dappe, ${ }^{\ddagger}$ and Jérôme Lagoute ${ }^{* \dagger}$ \\ ${ }^{\dagger}$ Laboratoire Matériaux et Phénomènes Quantiques, CNRS-Université Paris 7, 10 rue Alice Domon et Léonie Duquet, 75205 Paris \\ Cedex 13, France \\ ${ }^{\ddagger}$ SPEC, CEA, CNRS, Université Paris-Saclay, CEA Saclay, 91191 Gif-sur-Yvette Cedex, France
}

\section{Supporting Information}

ABSTRACT: Free-base porphyrin molecules offer appealing options to tune the interaction with their environment via the manipulation of their inner hydrogen atoms and molecular conformation. Using scanning tunneling microscopy we show, through a systematic study, that the molecular conformation, electronic gap, wave function, and molecule-substrate interaction are modified by hydrogen switch or removal. Experimental results in combination with ab initio calculations provide an understanding of the underlying physical process.

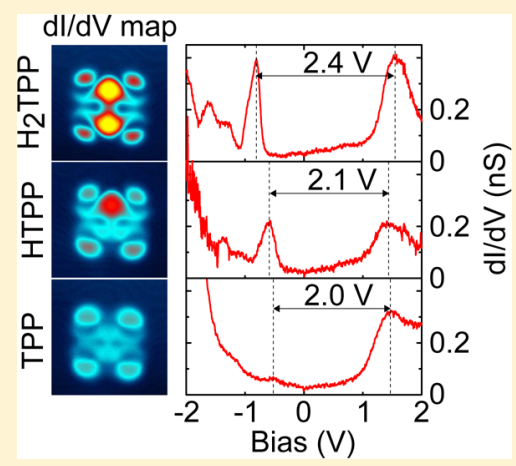

$\mathrm{P}$ oprhyrin molecules in contact with a supporting material have been widely studied in recent years because of their promising properties allowing the design of new functional materials. ${ }^{1,2}$ Among the series of derivatives, tetraphenyl porphyrins are excellent prototypes to study and exploit the properties of supported molecular macrocycles. Energy transfer, ${ }^{3-5}$ molecular switch, ${ }^{6}$ charge transfer, ${ }^{7-10}$ Kondo effect, ${ }^{9-12}$ conformational modification, ${ }^{1,13}$ and self-organization ${ }^{8,14,15}$ have been studied with such molecules adsorbed on metals or carbon materials. Tetraphenyl porphyrin was also proposed as a molecular logic gate exploiting the change of optical absorption spectrum under protonation or deprotonation. ${ }^{16}$ The possibility to manipulate individual molecules offered by scanning tunneling microscopy (STM) opens up a field of investigation of the interplay between molecular manipulation and interface process. Using the STM tip, it is possible to induce transfer (tautomerization) or removal (dehydrogenation) of hydrogen atoms. ${ }^{6,8,17-19}$ However, how these manipulation events modify the molecule-substrate interaction, the molecular spectrum, and conformation still has to be understood. Here, we provide a comprehensive study of the effect of tautomerization and dehydrogenation of a free-base porphyrin on its electronic states and interaction with the substrate. Combining scanning tunneling spectroscopy (STS) with ab initio calculations, we show how the manipulation of hydrogen atoms in a porphyrin molecule modifies the molecule-surface interaction. This modification is measured and analyzed through the molecular electronic gaps, the electronic wave functions, and the scattering of the substrate electronic states by the molecules.
After deposition of $\mathrm{H}_{2}$ TPP on $\mathrm{Au}(111)$, individual molecules, randomly distributed, can be seen on the surface. These molecules appear either with two bright protrusions in the center (Figure 1a) or no protrusion (Figure 1d) at $-0.8 \mathrm{~V}$. These shapes correspond to two tautomer forms that differ by the position of the inner hydrogen pair. ${ }^{6,8}$ In the following, $\mathrm{H}_{\alpha}$ denotes the conformation with two protrusions and $\mathrm{H}_{\kappa}$ denotes the conformation without protrusion following previous works. ${ }^{6,8}$ It is possible to reversibly switch between the two forms using an excitation by the tunneling current (see Supporting Information, Figure S1) as it was shown on $\operatorname{Ag}(111),{ }^{6} \operatorname{Au}(111),{ }^{8}$ and graphene. ${ }^{8}$ In contrast with the negative bias images, the topography at $+1.5 \mathrm{~V}$ does not reflect the hydrogen positions (Figure $1 \mathrm{~b}, \mathrm{e}$ ). This indicates that the shape of the molecules in the STM images is strongly affected by electronic effects. To deeply understand these features, we performed an extensive STS study. In Figure 1c,f, we report the $\mathrm{d} I / \mathrm{d} V$ spectra recorded above the macrocycle of the two molecules. The spectra reveal two resonances corresponding to the highest occupied molecular orbital (HOMO) at $-0.83 \mathrm{~V}$ and the lowest unoccupied molecular orbital (LUMO) at +1.55 $\mathrm{V}$. This leads to an electronic gap of $2.4 \mathrm{eV}$ corresponding to the gap of the free molecule reduced by the screening due to the substrate. ${ }^{8}$ The shape of the molecular states is revealed by the conductance maps reported in Figure la,b,d,e recorded at the bias of the HOMO and LUMO state peaks. The difference

Received: February 29, 2016

Accepted: March 30, 2016

Published: March 30, 2016 
HOMO

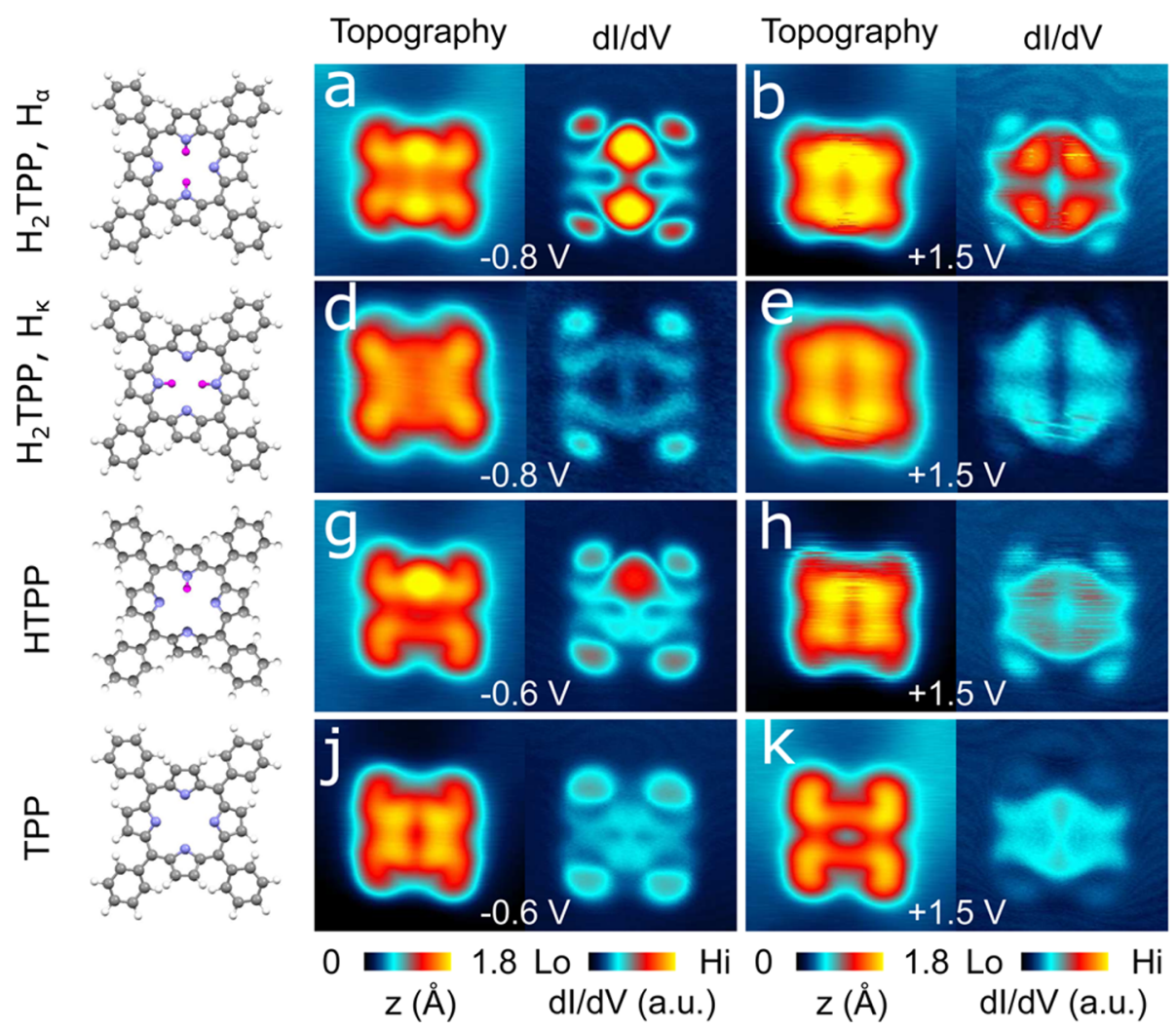

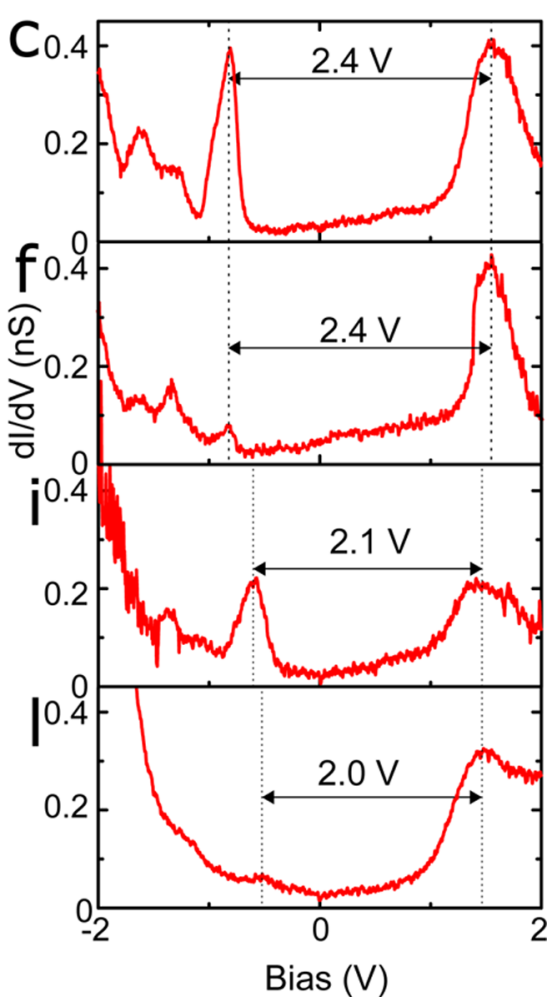

Figure 1. Topographic STM images and corresponding $d I / d V$ maps of $\mathrm{H}_{2} T P P$ in the $\mathrm{H}_{\alpha}(\mathrm{a}, \mathrm{b})$ and $\mathrm{H}_{\kappa}(\mathrm{d}, \mathrm{e})$ tautomer forms and $\mathrm{HTPP}(\mathrm{g}, \mathrm{h})$ and TPP $(j, k)$ at different bias voltages corresponding to the HOMO and LUMO states. $d I / d V$ spectra measured on $(c) \mathrm{H}_{2} \mathrm{TPP}\left(\mathrm{H}_{\alpha}\right)$, (f) $\mathrm{H}_{2} \mathrm{TPP}\left(\mathrm{H}_{\kappa}\right)$, (i) HTPP, and (l) TPP at a position corresponding to the bright lobe observed in the conductance map of HTPP.

between the HOMO state of the two tautomer forms is very striking in the conductance maps: two very bright lobes appear for $\mathrm{H}_{\alpha}$ and are absent for the $\mathrm{H}_{\kappa}$ configuration. This strong difference in the HOMO states of the two tautomer forms is at the origin of the contrast observed in the topography at the HOMO energy and will be explained below.

To explore the possibility of manipulating the molecular spectrum and controlling the molecule-substrate interaction, we performed deprotonation experiments induced by a voltage pulse. 6 By placing the tip above a molecule and applying a voltage pulse of $2.4 \mathrm{~V}$, we were able to remove one of the inner hydrogen atoms. The topography and electronic states of the resulting HTPP molecule are reported in Figure $1 \mathrm{~g}-\mathrm{i}$. The HOMO state of HTPP is shifted toward the Fermi level as compared to $\mathrm{H}_{2}$ TPP and appears at $-0.6 \mathrm{~V}$. The LUMO state appears at $+1.5 \mathrm{~V}$ which also reflects a shift toward the Fermi level but to a lower extent. The deprotonation therefore leads to a reduction of the electronic gap of the molecule. The respective intensities of the HOMO and LUMO peaks depend on the location where the spectrum is measured, as discussed in the Supporting Information (Figure S2). The HOMO state of HTPP now exhibits only one intense lobe (Figure 1g), which shows that the lobe is located around the position of the inner hydrogen atom. The LUMO state is not noticeably affected and still exhibits four lobes (Figure $1 \mathrm{~h}$ ).

To further understand the effect of hydrogen removal, we performed a second deprotonation that produces a TPP molecule. Panels $\mathrm{j}$ and $\mathrm{k}$ in Figure 1 display the topography of TPP at $-0.6 \mathrm{~V}$ and $+1.5 \mathrm{~V}$, respectively, that show no bright protrusion in the center of the molecule. The $\mathrm{d} I / \mathrm{d} V$ spectrum in Figure 11 shows that the HOMO peak appears only as a faint peak but is still measurable, and the HOMO-LUMO gap further reduces to $2.0 \mathrm{~V}$. These measurements show that successive deprotonations of $\mathrm{H}_{2} \mathrm{TPP}$ reduce progressively the molecular electronic gap. As we will discuss, this evolution is due to a change of the molecular structure and moleculesubstrate interaction. It is worth noting that a normalization procedure of the spectra can be applied, as discussed in the Supporting Information (Figure S3), and the same variation of the gap is observed in that case.

To understand these experimental observations, we performed $a b$ initio density functional theory (DFT) calculations for free and adsorbed molecules using the planewaves electronic structure package Quantum-Espresso (QE). ${ }^{20}$ The optimized structure of the molecules after adsorption (see Figure 2a,b) shows that, in addition to the rotation of the phenyl groups, in all cases two pyrrole groups point upward (i.e., carbon atoms higher than nitrogen with respect to the surface) while the two other pyrroles point downward (i.e., carbon atoms lower than nitrogen with respect to the surface). For the $\mathrm{H}_{\alpha}$ configuration, the angles of pyrrole groups pointing either up- or downward are about $30^{\circ}$, while the phenyl rings are rotated by about $20^{\circ}$. The $\mathrm{H}_{\kappa}$ configuration was found to be higher in energy by about $0.03 \mathrm{eV}$ as compared to the $\mathrm{H}_{\alpha}$ one.

After dehydrogenation, the pyrrole groups pointing upward rotate further by $10^{\circ}$, increasing their angle with the surface plane and approaching the nitrogen atom closer to the surface (the $\mathrm{N}-\mathrm{Au}$ distance reduces from 3.33 to $2.41 \AA$ ). Surprisingly, 
a
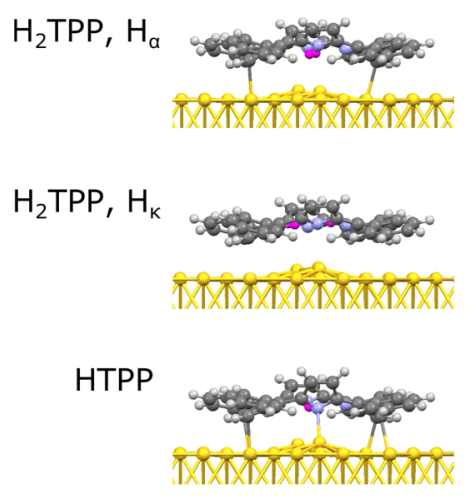

TPP

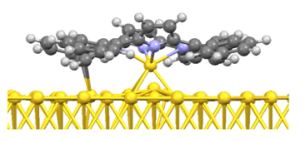

b
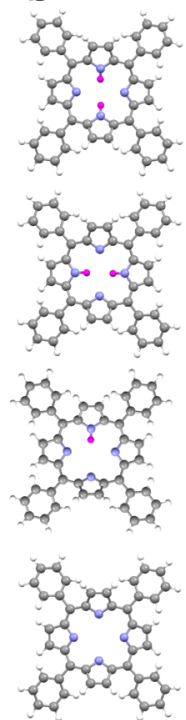

C HOMO
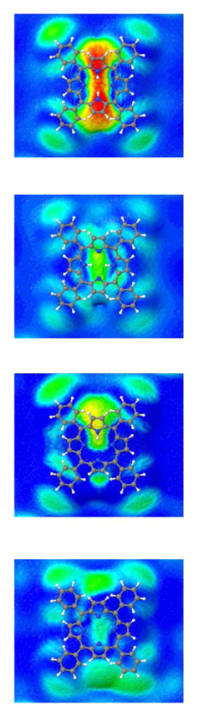
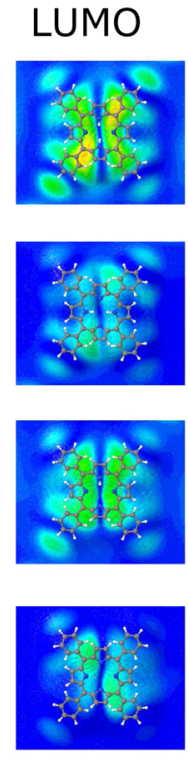

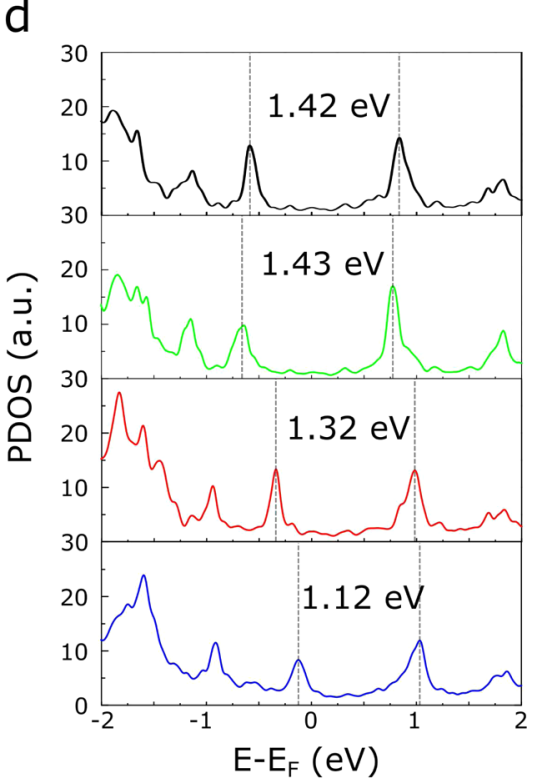

Figure 2. (a) Side view of the optimized structure of the molecules $\mathrm{H}_{2} \mathrm{TPP}\left(\mathrm{H}_{\alpha}\right.$ and $\left.\mathrm{H}_{\kappa}\right)$, HTPP, and TPP adsorbed on a Au(111) surface. (b) Top view of these molecules (gold atoms not shown). In panels a and b, nitrogen atoms are in light blue and inner hydrogens are marked in pink. (c) LDOS maps (on the topography surface taken at about $6 \AA$ above the molecule) for the four molecules at the energy of HOMO and LUMO peaks. Note the color code is different from that of the experimental images-from red (high) to blue (low). (d) Projected density of states (PDOS) on the molecule. The HOMO-LUMO gap is indicated in each case.

for the fully dehydrogenated molecule, we find that the $\mathrm{Au}$ atom of the surface located below the center of the molecule is lifted because of the interaction of the two nitrogen atoms of upward pyrroles. The distances with the four nitrogen atoms are 2.30, 2.59, 2.34, and $2.37 \AA$. This atom is therefore trapped between the molecule and the surface and is bounded both to the $\mathrm{Au}$ atoms of the surface and the nitrogen atoms of the molecule. The dehydrogenation reduces the molecule-surface distance as indicated by the height of the $\mathrm{C}$ atoms connecting phenyl and pyrrole rings with respect to the surface plane. This distance is $3.27,3.30,3.16$, and $3.14 \AA$ for $\mathrm{H}_{\alpha}, \mathrm{H}_{\kappa}, \mathrm{HTPP}$, and TPP, respectively.

The electronic density of states projected on the molecule (all atomic orbitals of the molecule) reported in Figure $2 \mathrm{~d}$ shows that the HOMO-LUMO gap reduces for consecutive dehydrogenation from $1.42 \mathrm{eV}$ (for $\mathrm{H}_{2} \mathrm{TPP}$ ) to $1.32 \mathrm{eV}$ (for HTPP) and $1.12 \mathrm{eV}$ (for TPP). The displacement of the HOMO level toward the Fermi level of the interface upon dehydrogenation reflects the electron transfer toward the surface that increases with dehydrogenation. As it is wellknown in DFT, the calculated gap is underestimated with respect to experiments. This general drawback of the DFT can explain that calculated LUMO positions are found to be lower in energy by about $0.5 \mathrm{eV}$ as compared to corresponding STS features. However, we can reproduce the tendency of gap reduction with dehydrogenation, as observed experimentally. To estimate the contribution of the substrate in this effect, we have calculated the spectra of the molecules with the same conformation but without substrate. The resulting HOMOLUMO gaps are $1.42,1.33$, and $1.33 \mathrm{eV}$, respectively. This shows that the successive gap reduction results from a combination of an intrinsic reduction in the spectrum of the molecule itself (induced by hydrogen removal and conformational change) and an electronic interaction with the substrate. Note that the intrinsic reduction involves the conformational change that is also induced by the interaction with the substrate. It is worth noting that a gap reduction induced by a change of molecular conformation has been evidenced previously by Müllegger et al. ${ }^{21}$ for AuTPP molecules adsorbed on $\mathrm{Au}(111)$. The authors found that different conformations of the AuTPP molecules coexist on the surface and that when the asymmetry increases the molecule gap reduces. Such a conformational effect is partly responsible for the gap variations that we observe, together with the dehydrogenation and change of molecule-substrate interaction.

We have simulated theoretically the STS conductance maps by calculating the local density of states (LDOS) at the energy of interest (applied voltage) on the iso-surface of LDOS integrated from the Fermi level to that energy. The latter simulates the constant current STM topography whose nonflat shape may affect the details of conductance maps measured experimentally. Figure $2 \mathrm{c}$ presents such maps at the energy of the HOMO and LUMO peak for each molecule. For the LUMO state, four lobes centered on the side part of pyrroles with minima located at the centers of pyrroles are evidenced in agreement with the experiment. For $\mathrm{H}_{2}$ TPP, these four lobes are independent of the position of the hydrogen atoms as can be seen from the comparison between $\mathrm{H}_{\alpha}$ and $\mathrm{H}_{\kappa}$ configurations in Figure 2c. No substantial change is observed in the LUMO state of the HTPP and TPP molecule, which is also in agreement with the experiment. The LUMO state is therefore not sensitive to the inner hydrogen location. Note that the LUMO feature is in fact a superposition of two nearly degenerate states, LUMO1 and LUMO2, that have a small contribution on inner $\mathrm{H}$ atoms (see Supporting Information, Figure S4). On another side, for the HOMO state, the surprisingly large difference between the two tautomer forms of $\mathrm{H}_{2}$ TPP observed in the experiment is reproduced in the calculations, as shown in Figure 2c. For the $\mathrm{H}_{\alpha}$ form, two bright lobes appear above the hydrogenated pyrrole groups that point upward. For the $\mathrm{H}_{\kappa}$ form, these lobes disappear, which corresponds exactly to the experimental observation. Actually, 
there are still two lobes at the pyrrole groups at which the hydrogen atoms are linked, but they are pointing toward the surface and mix with the surface states. In the STM experiment, as we probe the top side of the molecule, these lobes, which are located below the molecule, cannot be observed. This is why we observe such a strong variation of the shape of HOMO for the two tautomer forms of $\mathrm{H}_{2} \mathrm{TPP}$ in the STM images. Furthermore, the mixing of the lobes with the surface states enhances the hybridization of HOMO with the substrate, which can be seen from the broader HOMO peak in the PDOS with respect to the $\mathrm{H}_{\alpha}$ configuration.

In the case of the HTPP molecule, the LDOS map confirms that a lobe is observed only on the pyrrole group with a hydrogen atom. Interestingly, another pyrrole group (without a $\mathrm{H}$ atom) pointing upward is even higher, by about $0.45 \AA$, because of its additional rotation induced by the binding of the $\mathrm{N}$ atom to the surface discussed above. However, no bright spot is seen above this pyrrole, reflecting the purely electronic and not geometrical origin of the pyrrole lobes of the conductance maps. This is further confirmed by the calculated LDOS of TPP where no bright lobe is observed although two pyrrole groups are pointing upward. In addition, structural modifications of the system modify the electronic interaction between the molecule and the surface. As can be seen in Figure $2 \mathrm{a}$, consecutive dehydrogenations induce bound formations between the molecule and the surface. In the case of HTPP, some carbon atoms of a down pyrrole and the nitrogen atom for which hydrogen has been removed are bounded to the surface. In the case of TPP, the molecule-surface interaction increases so much that a gold atom of the surface shifts to an intermediate position between the surface and the molecule. The picking up of a substrate atom by a $\mathrm{H}_{2}$ TPP molecule has been previously observed on iron and nickel surfaces. ${ }^{22}$ More recently, a self-metalation on an inert substrate has been evidenced. $^{23}$ Our data suggest that TPP molecules can also strongly interact with a $\mathrm{Au}(111)$ surface such that a gold atom of the surface can be lifted toward the center of the macrocyle. The HOMO-LUMO gap of the TPP molecule interacting with the surface is reduced as compared to $\mathrm{H}_{2}$ TPP and HTPP. The interaction of $\mathrm{H}_{2}$ TPP with a $\mathrm{Au}$ adatom on a $\mathrm{Au}(111)$ surface has been recently reported, ${ }^{24}$ showing that the interaction of the molecule with the adatom induces rigid shift of the molecular-induced states to higher energies. In the present case, the combination of dehydrogenation and interaction of the molecule with a $\mathrm{Au}$ atom of the substrate leads to a reduction in the molecular gap.

To probe the interaction of the three hydrogenation degrees of porphyrins with the surface, we have measured the scattering of the Shockley surface state induced by the molecules that can provide information on the molecule-surface coupling. ${ }^{25}$ Figure 3 shows a series of conductance maps revealing the electron standing wave patterns resulting from the scattering of the surface state by the molecules.

The three examples correspond to the same molecule that was successively deprotonated in order to compare the surface state scattering by $\mathrm{H}_{2}$ TPP, HTPP, and TPP. As can be seen, the amplitude of the standing wave pattern increases after one and two deprotonation (see also Supporting Information, Figure S5) indicating a more efficient scattering of the deprotonated molecules and therefore a larger electronic interaction between the molecule and the substrate, which is in very good agreement with the broadening of the electronic states and the reduction of the HOMO-LUMO gap discussed above.

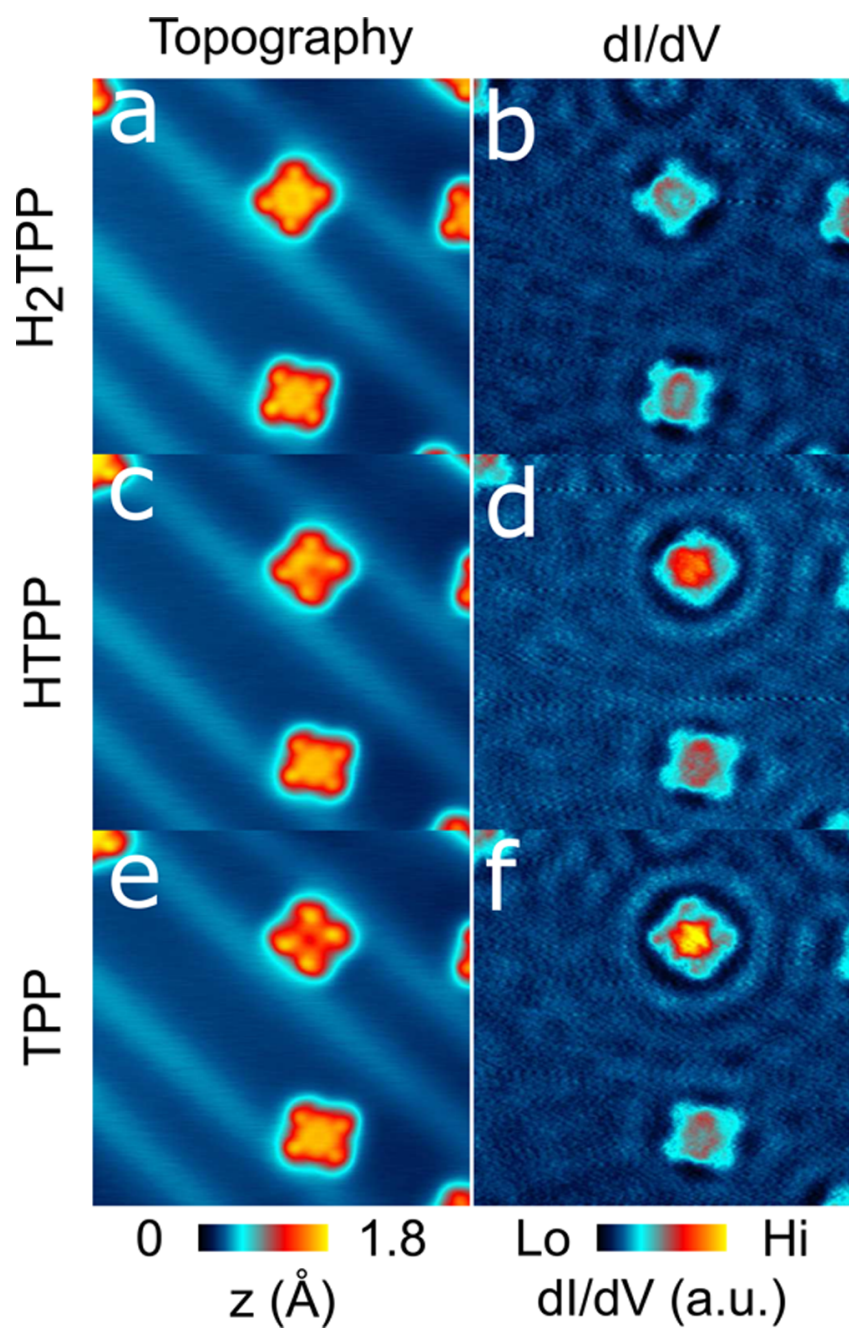

Figure 3. STM topography image $\left(10 \times 10 \mathrm{~nm}^{2}, 0.8 \mathrm{~V}, 1 \mathrm{nA}\right)$ of $\mathrm{H}_{2}$ TPP (a), HTPP (c), and TPP (e) and corresponding conductance maps at $0.8 \mathrm{~V}(\mathrm{~b}, \mathrm{~d}, \mathrm{f})$.

In summary, the electronic properties of the free-base porphyrins $\mathrm{H}_{2}$ TPP adsorbed on $\mathrm{Au}(111)$ manipulated with an STM tip have been analyzed. The molecule can be switched between its two tautomer forms using the tunneling current. The HOMO state of these forms can display two bright lobes $\left(\mathrm{H}_{\alpha}\right.$ form $)$ or no lobe $\left(\mathrm{H}_{\kappa}\right.$ form $)$. We have shown that in all cases the wave function presents two lobes on the hydrogenated pyrrole group; however, for the $\mathrm{H}_{\kappa}$ form, these lobes are turned toward the surface and therefore are not observed in experimental STM images. Other forms of porphyrin were produced by tip-induced dehydrogenation. We showed that the conformational change and the interaction with the surface progressively reduces the HOMO-LUMO gap when one and two hydrogen are removed. Dehydrogenation was also shown to increase the molecule-surface interaction, leading to increased scattering of the Shockley surface state of $\mathrm{Au}(111)$ until the binding of a surface gold atom by the central part of the dehydrogenated molecule. These results provide a comprehensive data set and physical understanding of the properties of different forms of free-base molecules in contact with a supporting substrate. The possibility to switch between different forms after adsorption of the molecules offers possibilities to tune the molecule-substrate interface in such systems. 


\section{EXPERIMENTAL SECTION}

All the experiments were performed using a STM instrument (Omicron) operating in ultrahigh vacuum (UHV) at a pressure below $1 \times 10^{-10} \mathrm{mbar}$ and at a temperature of $4.6 \mathrm{~K}$. Au(111) on mica substrate was prepared by repeated $\mathrm{Ar}^{+}$sputtering (900 $\mathrm{eV})$ and annealing $(600 \mathrm{~K})$ under UHV conditions to obtain an atomically clean surface. 5,10,15,20-Tetraphenyl-21H,23Hporphyrin $\left(\mathrm{H}_{2} \mathrm{TPP}\right)$ molecules (Aldrich) were deposited on $\mathrm{Au}(111)$ surface using an effusion cell (Dr. Eberl MBEKomponenten $\mathrm{GmbH}$ ) under vacuum at $525 \mathrm{~K}$ at the STM stage maintained at $4.6 \mathrm{~K}$. All the STM images were recorded in constant-current mode with an electrochemically etched tungsten tip. The $\mathrm{d} I / \mathrm{d} V$ spectra were acquired using a lock-in detector at a frequency of ca. $670 \mathrm{~Hz}$ and a modulation amplitude of $35 \mathrm{mV}$.

The DFT calculations were carried out using generalized gradient approximation (GGA) in the Perdew, Burke, and Ernzerhof parametrization (PBE) for exchange-correlation functionals. The whole system was simulated by three $\mathrm{Au}(111)$ layers with the molecule adsorbed on one side, and a $(9 \times 9)$ in-plane supecell was employed to avoid artificial molecule-molecule interactions. During atomic relaxation, two $\mathrm{Au}$ layers were kept fixed while the molecule and the surface layer in contact with it were allowed to relax until atomic forces became lower than $10^{-3} \mathrm{Ry} / \mathrm{bohr}$. We also took partially into account van der Waals interactions using semiempirical dispersion corrections (DFT-D) as formulated by Grimme ${ }^{26}$ and implemented in the $\mathrm{QE}$ package. The relaxation were performed using only $\Gamma$ k-point, while for electronic structure analysis, a more dense $(4 \times 4) \mathrm{k}$-point mesh was employed. The plane wave cutoff energies of 30 and 300 Ry were used for electronic wave functions and charge density, respectively.

\section{ASSOCIATED CONTENT}

\section{S Supporting Information}

The Supporting Information is available free of charge on the ACS Publications website at DOI: 10.1021/acs.jpclett.6b00476.

Tip-induced switching of inner hydrogen atoms in $\mathrm{H}_{2}$ TPP molecules, position-dependent spectra above $\mathrm{H}_{2}$ TPP molecules, normalization of $\mathrm{d} I / \mathrm{d} V$ spectra, molecule-projected density of states, and surface state scattering (PDF)

\section{AUTHOR INFORMATION}

\section{Corresponding Author}

*E-mail: jerome.lagoute@univ-paris-diderot.fr.

\section{Notes}

The authors declare no competing financial interest.

\section{ACKNOWLEDGMENTS}

ANR (Agence Nationale de la Recherche) and CGI (Commissariat á l'Investissement d'Avenir) are gratefully acknowledged for their financial support of this work through Labex SEAM (Science and Engineering for Advanced Materials and devices) ANR 11 LABX 086, ANR 11 IDEX 05 02. V.R. thanks the Institut Universitaire de France for support. The calculations have been performed using HPC computation resources from GENCI-[TGCC] (Grant 2016097416).

\section{REFERENCES}

(1) Jurow, M.; Schuckman, A. E.; Batteas, J. D.; Drain, C. M. Porphyrins as Molecular Electronic Components of Functional Devices. Coord. Chem. Rev. 2010, 254, 2297-2310.

(2) Auwärter, W.; Écija, D.; Klappenberger, F.; Barth, J. V. Porphyrins at Interfaces. Nat. Chem. 2015, 7, 105-120.

(3) Roquelet, C.; Lauret, J.-S.; Alain-Rizzo, V.; Voisin, C.; Fleurier, R.; Delarue, M.; Garrot, D.; Loiseau, A.; Roussignol, P.; Delaire, J. A.; et al. Pi-Stacking Functionalization of Carbon Nanotubes through Micelle Swelling. ChemPhysChem 2010, 11, 1667-1672.

(4) Roquelet, C.; Garrot, D.; Lauret, J.-S.; Voisin, C.; Alain-Rizzo, V.; Roussignol, P.; Delaire, J.; Deleporte, E. Quantum Efficiency of Energy Transfer in Noncovalent Carbon Nanotube/Porphyrin Compounds. Appl. Phys. Lett. 2010, 97, 141918.

(5) Roquelet, C.; Vialla, F.; Diederichs, C.; Roussignol, P.; Delalande, C.; Deleporte, E.; Lauret, J.-S.; Voisin, C. Local Field Effects in the Energy Transfer between a Chromophore and a Carbon Nanotube: A Single-Nanocompound Investigation. ACS Nano 2012, 6, 8796-8802.

(6) Auwärter, W.; Seufert, K.; Bischoff, F.; Ecija, D.; Vijayaraghavan, S.; Joshi, S.; Klappenberger, F.; Samudrala, N.; Barth, J. V. A SurfaceAnchored Molecular Four-Level Conductance Switch Based on Single Proton Transfer. Nat. Nanotechnol. 2011, 7, 41-46.

(7) Dyer, M. S.; Robin, A.; Haq, S.; Raval, R.; Persson, M.; Klimeš, J. Understanding the Interaction of the Porphyrin Macrocycle to Reactive Metal Substrates: Structure, Bonding, and Adatom Capture. ACS Nano 2011, 5, 1831-1838.

(8) Pham, V. D.; Lagoute, J.; Mouhoub, O.; Joucken, F.; Repain, V.; Chacon, C.; Bellec, A.; Girard, Y.; Rousset, S. Electronic Interaction between Nitrogen-Doped Graphene and Porphyrin Molecules. ACS Nano 2014, 8, 9403-9409.

(9) Kim, H.; Chang, Y. H.; Lee, S.-H.; Kim, Y.-H.; Kahng, S.-J. Switching and Sensing Spin States of Co-Porphyrin in Bimolecular Reactions on $\mathrm{Au}(111)$ Using Scanning Tunneling Microscopy. ACS Nano 2013, 7, 9312-9317.

(10) Schouteden, K.; Ivanova, T.; Li, Z.; Iancu, V.; Janssens, E.; Van Haesendonck, C. Probing Magnetism in 2D Molecular Networks after in Situ Metalation by Transition Metal Atoms. J. Phys. Chem. Lett. 2015, 6, 1048-1052.

(11) Iancu, V.; Deshpande, A.; Hla, S.-W. Manipulating Kondo Temperature via Single Molecule Switching. Nano Lett. 2006, 6, 820823.

(12) Wang, W.; Pang, R.; Kuang, G.; Shi, X.; Shang, X.; Liu, P. N.; Lin, N. Intramolecularly Resolved Kondo Resonance of High-Spin Fe (II)-Porphyrin Adsorbed on Au (111). Phys. Rev. B: Condens. Matter Mater. Phys. 2015, 91, 045440.

(13) Moresco, F.; Meyer, G.; Rieder, K.-H.; Tang, H.; Gourdon, A.; Joachim, C. Conformational Changes of Single Molecules Induced by Scanning Tunneling Microscopy Manipulation: A Route to Molecular Switching. Phys. Rev. Lett. 2001, 86, 672-675.

(14) Bussetti, G.; Campione, M.; Riva, M.; Picone, A.; Raimondo, L.; Ferraro, L.; Hogan, C.; Palummo, M.; Brambilla, A.; Finazzi, M.; et al. Stable Alignment of Tautomers at Room Temperature in Porphyrin 2D Layers. Adv. Funct. Mater. 2014, 24, 958-963.

(15) Ruggieri, C.; Rangan, S.; Bartynski, R. A.; Galoppini, E. Zinc (II) Tetraphenylporphyrin Adsorption on Au (111): An Interplay Between Molecular Self-Assembly and Surface Stress. J. Phys. Chem. C 2015, $119,6101-6110$.

(16) Langford, S. J.; Yann, T. Molecular Logic: A Half-Subtractor Based on Tetraphenylporphyrin. J. Am. Chem. Soc. 2003, 125, 1119811199.

(17) Liljeroth, P.; Repp, J.; Meyer, G. Current-Induced Hydrogen Tautomerization and Conductance Switching of Naphthalocyanine Molecules. Science 2007, 317, 1203-1206.

(18) Bischoff, F.; Seufert, K.; Auwa rter, W.; Joshi, S.; Vijayaraghavan, S.; Ecija, D.; Diller, K.; Papageorgiou, A. C.; Fischer, S.; Allegretti, F.; et al. How Surface Bonding and Repulsive Interactions Cause Phase Transformations: Ordering of a Prototype Macrocyclic Compound on Ag (111). ACS Nano 2013, 7, 3139-3149. 
(19) Kumagai, T.; Hanke, F.; Gawinkowski, S.; Sharp, J.; Kotsis, K.; Waluk, J.; Persson, M.; Grill, L. Thermally and Vibrationally Induced Tautomerization of Single Porphycene Molecules on a $\mathrm{Cu}$ (110) Surface. Phys. Rev. Lett. 2013, 111, 246101.

(20) Giannozzi, P.; Baroni, S.; Bonini, N.; Calandra, M.; Car, R.; Cavazzoni, C.; Ceresoli, D.; Chiarotti, G. L.; Cococcioni, M.; Dabo, I. e. a. QUANTUM ESPRESSO: a Modular and Open-Source Software Project for Quantum Simulations of Materials. J. Phys.: Condens. Matter 2009, 21, 395502.

(21) Müllegger, S.; Rashidi, M.; Lengauer, T.; Rauls, E.; Schmidt, W.; Knör, G.; Schöfberger, W.; Koch, R. Asymmetric Saddling of Single Porphyrin Molecules on Au (111). Phys. Rev. B: Condens. Matter Mater. Phys. 2011, 83, 165416.

(22) Goldoni, A.; Pignedoli, C. A.; Di Santo, G.; Castellarin-Cudia, C.; Magnano, E.; Bondino, F.; Verdini, A.; Passerone, D. Room Temperature Metalation of $2 \mathrm{H}$-TPP Monolayer on Iron and Nickel Surfaces by Picking up Substrate Metal Atoms. ACS Nano 2012, 6, 10800-10807.

(23) Smykalla, L.; Shukrynau, P.; Mende, C.; Lang, H.; Knupfer, M.; Hietschold, M. Photoelectron Spectroscopy Investigation of the Temperature-Induced Deprotonation and Substrate-Mediated Hydrogen Transfer in a Hydroxyphenyl-Substituted Porphyrin. Chem. Phys. 2015, 450-451, 39-45.

(24) Mielke, J.; Hanke, F.; Peters, M. V.; Hecht, S.; Persson, M.; Grill, L. Adatoms underneath Single Porphyrin Molecules on $\mathrm{Au}$ (111). J. Am. Chem. Soc. 2015, 137, 1844-1849.

(25) Gross, L.; Moresco, F.; Savio, L.; Gourdon, A.; Joachim, C.; Rieder, K.-H. Scattering of Surface State Electrons at Large Organic Molecules. Phys. Rev. Lett. 2004, 93, 056103.

(26) Grimme, S. Semiempirical GGA-type Density Functional Constructed with a Long-Range Dispersion Correction. J. Comput. Chem. 2006, 27, 1787-1799. 